Portland State University

PDXScholar

Physics Faculty Publications and Presentations

Physics

3-1-2010

\title{
Correction of dark current in consumer cameras
}

Justin Charles Dunlap

Portland State University

Erik Bodegom

Portland State University

Ralf Widenhorn

Portland State University

Follow this and additional works at: https://pdxscholar.library.pdx.edu/phy_fac

Part of the Physics Commons

Let us know how access to this document benefits you.

\section{Citation Details}

Justin C. Dunlap, Erik Bodegom and Ralf Widenhorn "Correction of dark current in consumer cameras", J. Electron. Imaging. 19(1), 013010 (2010).

This Article is brought to you for free and open access. It has been accepted for inclusion in Physics Faculty Publications and Presentations by an authorized administrator of PDXScholar. Please contact us if we can make this document more accessible: pdxscholar@pdx.edu. 


\title{
Correction of dark current in consumer cameras
}

\author{
Justin C. Dunlap \\ Erik Bodegom \\ Ralf Widenhorn \\ Portland State University \\ P.O Box 751 \\ Portland, Oregon 97207-0751 \\ E-mail: ralfw@pdx.edu
}

\begin{abstract}
A study of dark current in digital imagers in digital singlelens reflex (DSLR) and compact consumer-grade digital cameras is presented. Dark current is shown to vary with temperature, exposure time, and ISO setting. Further, dark current is shown to increase in successive images during a series of images. DSLR and compact consumer cameras are often designed such that they are contained within a densely packed camera body, and therefore the digital imagers within the camera frame are prone to heat generated by the sensor as well as nearby elements within the camera body. It is the scope of this work to characterize the dark current in such cameras and to show that the dark current, in part due to heat generated by the camera itself, can be corrected by using hot pixels on the imager. This method generates computed dark frames based on the dark current indicator value of the hottest pixels on the chip. We compare this method to standard methods of dark current correction. () 2010 SPIE and IS\&T. [DOI: 10.1117/1.3358365]
\end{abstract}

\section{Introduction}

We have previously shown that noise generated by thermally excited electrons, so-called dark noise, in digital imagers can be corrected by analyzing the pixels that show the greatest amounts of dark current. This method has shown to work in general for charge-coupled devices ${ }^{1}$ (CCDs) as well as for complimentary metal-oxide semiconductor (CMOS) imagers. ${ }^{2}$ However, those studies had been carried out on scientific-grade imagers where the temperature of the imager can be carefully controlled, or in imagers outside of a camera body. This work demonstrates that the same method can be used to analyze and correct dark current for both CCD and CMOS imagers within digital single-lens reflex (DSLR) and compact consumer cameras.

The measurement of dark current in consumer cameras is obfuscated by inconsistent internal temperature within the camera body and by camera manufacturers' own efforts to deal with dark noise. As dark current is one of the largest sources of noise in digital imagers, camera manufacturers implement their own systems for removing the noise to provide better pictures at longer exposure times. Most commonly for images at long exposures, a second image with a closed shutter will be taken after the initial image. This second image is a dark frame, an image with no light signal, which is subtracted from the initial image to remove the dark noise, and will ideally leave only the light infor-

Paper 09221P received Nov. 17, 2009; accepted for publication Jan. 19, 2010; published online Mar. 12, 2010.

1017-9909/2010/19(1)/013010/11/\$25.00 @ 2010 SPIE and IS\&T. mation. However, there are problems associated with this method of correction. Foremost, this method is time consuming, as it is required after every exposure. Furthermore, the camera will not be under identical conditions for the second frame due to the generation of the first image itself. As is shown in this work, the dark current is not consistent from frame to frame and shows increasing dark current with increasing number of frames taken in succession. This is likely due to an increase in the internal temperature of the camera. In addition, due to the statistical nature of the dark current, even dark frames taken under identical conditions will not necessarily have the same amount of dark noise.

The very attributes that define the compact consumer class of camera, their affordability and compact size, are likely indicators that dark noise is a greater issue in this class of camera. Dark noise is caused by defects and impurities within the silicon of the pixel. ${ }^{3-5}$ The price point of compact consumer cameras would seem to indicate that the best digital imagers, in terms of lowest impurities and therefore lowest dark noise, would be selectively chosen for more expensive, higher-end cameras. Consumer cameras are generally not cooled, and therefore heat will be trapped until it can dissipate through the camera body; this was found to take at least a few minutes for the DSLR cameras we studied. Compact consumer cameras have the various electronic elements of the camera confined to an even smaller volume, suggesting a greater influence of heat generated by the various elements on the imager, and therefore also a greater effect on dark noise.

To accurately study the dark noise in the cameras, it is necessary to obtain images that have not been compressed nor processed. The typical file generated by consumer cameras is in the JPEG format, a format that allows for variable levels of compression. However, the compression is not lossless and some information, including some of the dark noise signal, is lost in the compression routines. In addition, many cameras will process the information from the imager, such as by sharpening or color correcting the image, in an effort to make the image more aesthetically pleasing. These processing routines further obscure the original signal from the imager and further mask the dark noise. DSLR models do allow the user to save images in a RAW file format that does not suffer from loss of information due to compression. However, the compact consumer models typically do not. To obtain uncompressed, unprocessed images 
in the RAW format, we used an open-source software program called CHDK, which is designed to be used with Canon cameras. ${ }^{6}$ In addition, CHDK allows for long exposure time control. Unfortunately, this limited the study of the compact consumer models to one manufacturer of cameras, Canon.

Two of the DSLR cameras studied, the Nikon D70 and Nikon D700, appear to have a median filter applied to the image after collection, regardless of whether the noise reduction mode is turned on or not. A median filter removes values of pixels with inconsistently large values, as compared with their neighbors, by applying an average or median value of those neighboring pixels to the unusually high valued pixels. The noise reduction mode appears to deal with dark noise by taking a second image as described in the previous paragraph. It was found that by enabling the noise reduction mode and turning the camera off before it was able to collect the second image, no median filter would be applied to the image and the dark current in the first image was saved.

While camera manufacturers do deal with the problem of dark noise in their cameras, they do not necessarily do the best possible job. In addition to studying the behavior of the dark current, we also attempt to improve on the dark noise correction methods used by the camera manufacturers by using the method of analyzing the hot pixels as dark current indicators for the entire image.

\section{Dark Current Behavior}

Data were gathered from four DSLR models and three compact consumer-grade cameras. Data are presented for one DSLR camera, the Canon EOS-20D, and one compact consumer camera, the Canon Powershot SD1000. In addition to the data presented for the Canon EOS-20D, dark current data for a Nikon D70, a Nikon D700, and a Canon EOS-20Da were taken and analyzed. These cameras all generate 12-bit pixel values except for the Nikon D700, which has a 14-bit pixel depth. In addition to the data presented for the SD1000, dark current from a Canon S2IS and a Canon PowerShot A570 IS, both compact consumer cameras, were analyzed. These cameras all generate images with a 10-bit pixel depth. Images were taken in a temperature-controlled, light-sealed enclosure. The cameras were allowed to reach thermal equilibrium with the air inside the box prior to obtaining images, and the temperature was monitored inside the enclosure throughout the imagetaking process. Data for one camera in each class are displayed next. The dark current behavior for each camera was unique; however, the broad results of predictable dark current with changes to temperature, ISO setting, exposure time, and increasing exposure number within a set of data are applicable to all of the cameras examined.

\subsection{Canon EOS-20D}

The Canon EOS-20D, introduced 19 August 2004, has a $22.5 \times 15.0-\mathrm{mm}$ CMOS sensor, with 8.2 million effective pixels in a grid of $3522 \times 2348$ pixels. To initially gauge the behavior of dark current in the Canon 20D, we collected images at varying exposure times starting at $30 \mathrm{~s}$, decreasing in 5-s intervals to $5 \mathrm{~s}$. Ten frames were taken at each exposure time and an average was taken for each pixel. The camera was set to an ISO setting of 1600, and

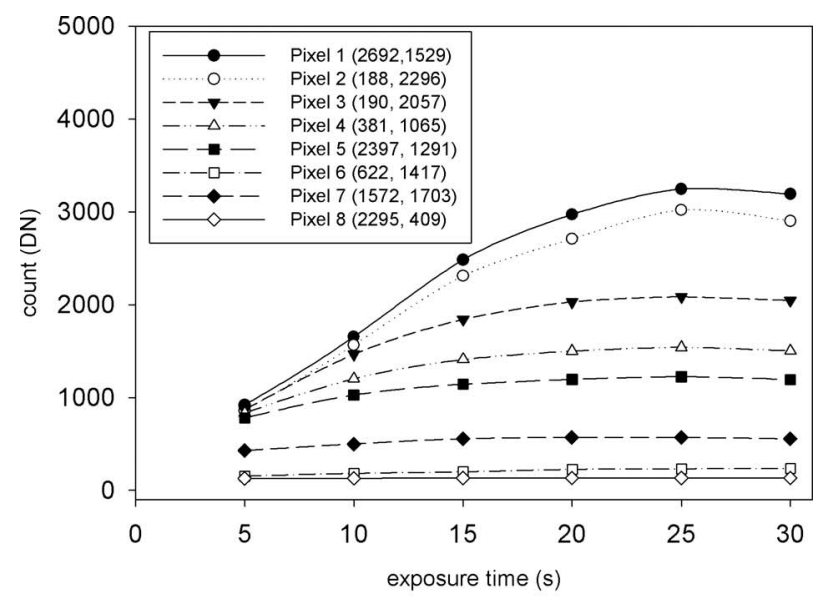

(a)

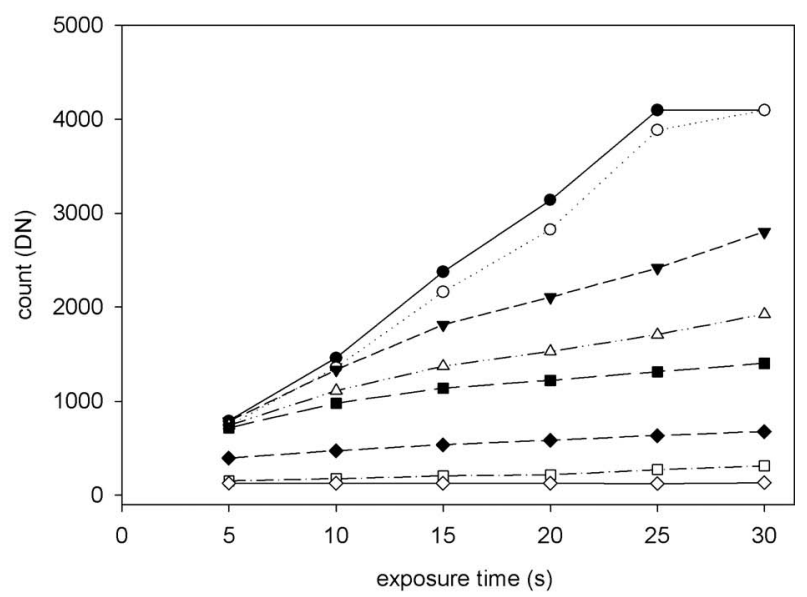

(b)

Fig. 1 Exposure time dependence of dark current for eight Canon 20D pixels, taken at ISO $=1600$ and $24^{\circ} \mathrm{C}$. (a) 30-s images taken first, decreasing to 5-s images. (b) 5-s images taken first, increasing to 30-s images. (Pixels shown are the same for both panels.)

the external temperature within the enclosure was $24^{\circ} \mathrm{C}$, with a temperature increase within the enclosure of approximately a degree for the entire run. Figure 1(a) shows the response of eight pixels on the imager. Pixel coordinates are given in parentheses for each pixel number. Pixels 1 through 7 are hot pixels to varying degrees, and pixel 8 is an example of a typical pixel on the imager that appears to have little dark current. After $20 \mathrm{~s}$, there appears to be a flattening of counts, with additional length in exposure time resulting in marginal change in dark counts for all pixels. As shown in Fig. 1(b), when the exposures were taken such that the 5-s exposures were taken first and then by increasing intervals of 5 to $30 \mathrm{~s}$, the counts no longer leveled off after $20 \mathrm{~s}$. Pixel 1 is saturated after $25 \mathrm{~s}$ at the maximum count and therefore does not show an increase in counts from 25 to $30 \mathrm{~s}$. An increase in internal temperature of the imager with each additional frame would result in more dark current in images taken later on in the run. This would affect the exposure time trend by apparently decreasing values for the exposure time taken first, and could account for the trend seen in Fig. 1(a).

Shown in Fig. 2 are the counts for eight pixels in the 


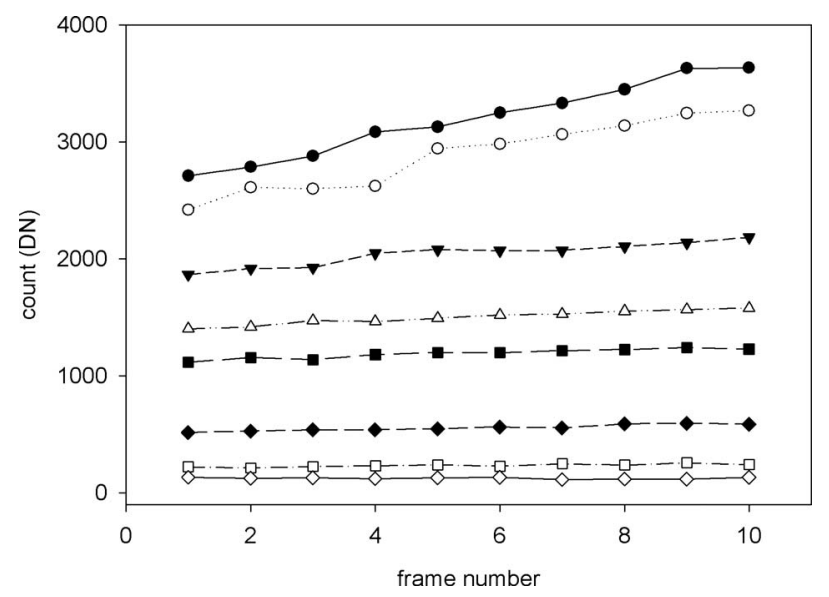

Fig. 2 Dark current dependence on frame numbers of eight pixels for ten 30-s frames separated by $5 \mathrm{~s}$ between frames taken at $I S O=1600$ and $24^{\circ} \mathrm{C}$. (Same pixels as shown in Fig. 1.)

30-s frames used to find the average in Fig. 1(a). For each pixel there is an increase in counts for each successive frame, with an increase of a third or more from the first frame to the last. This increase in value further lends evidence to the possibility that the internal temperature within the camera, and of the chip itself, increases significantly from each frame to the next. As discussed in the introduction in Sec. 1, a common method for correcting for dark current is to take a dark frame immediately after the initial exposure. This method assumes similar conditions for the two frames; however, from Fig. 2 it is evident that the act of obtaining a frame has changed the condition of the imager, and the second frame will likely have more dark noise than the initial frame.

To further study the dependence of dark noise on preceding frames, we took 100 frames each with an integration time of $30 \mathrm{~s}$ with $5 \mathrm{~s}$ between frames. The temperature within the enclosure started at $24.0^{\circ} \mathrm{C}$ and increased to $25.2{ }^{\circ} \mathrm{C}$ at the one hundredth frame. The hot pixels shown in Fig. 3 show a large increase in dark noise from the first frame to the last. The increase of $1.2^{\circ} \mathrm{C}$ in external tem-

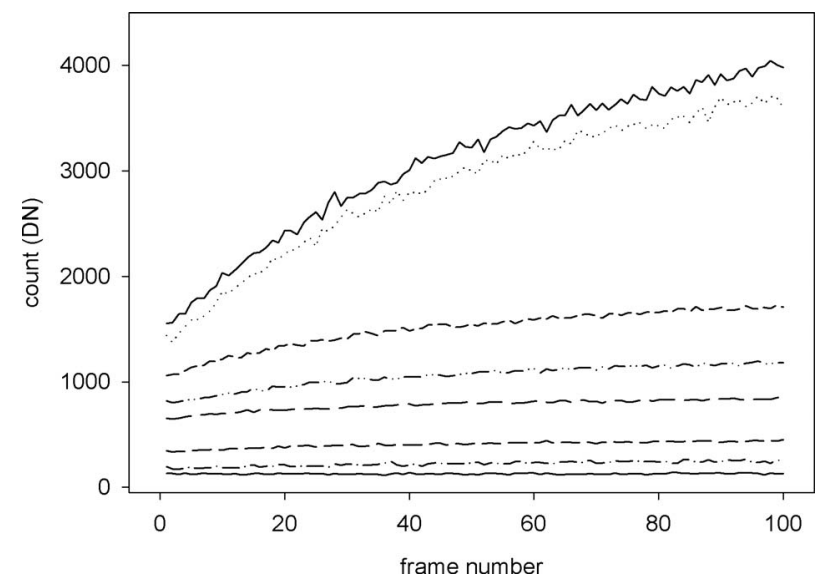

Fig. 3 Dependence of dark current for eight pixels on frame numbers for 100 frames with an integration time of $30 \mathrm{~s}$ and a rest period of $5 \mathrm{~s}$ between frames at ISO $=1600$. (Same pixels as shown in Fig. 1.)

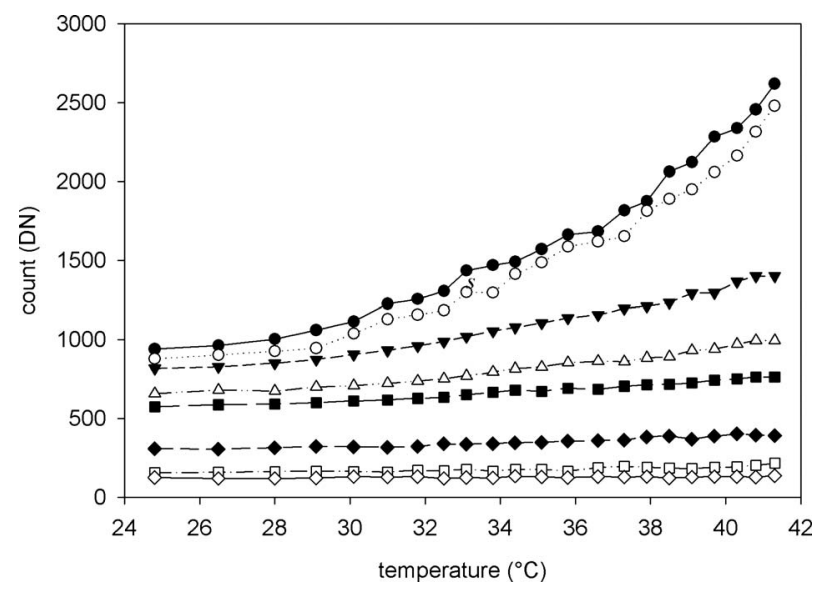

Fig. 4 Dependence of dark current for eight pixels on external temperature. 20-s exposures taken with an ISO setting of 800 . (Same pixels as shown in Fig. 1.)

perature is not large enough to account for such changes in dark noise, as is shown next. This again indicates that the internal temperature of the camera and the temperature of the sensor itself likely increase more dramatically than the external temperature. In a series of multiple frames with a rest period between sets of ten, it appeared, for this specific camera, to take a rest period of two to three minutes for the pixel values to return to where they started in the first frame.

A series of exposures were taken in an attempt to more accurately determine the pixels' response to temperature. The Canon 20D was placed within the enclosure with an adjustable heater. The heater was set to maintain a steady rise in temperature over a span of 105 min from a starting temperature of $24.8^{\circ} \mathrm{C}$ to the final temperature of $41.3^{\circ} \mathrm{C}$. Exposures lasting $20 \mathrm{~s}$ were taken every five minutes at $\mathrm{ISO}=800$. Shown in Fig. 4 is the response of eight pixels for this series. As is evident from the temperature response of the pixels shown in Fig. 4, the extreme increase in dark counts shown in Fig. 3 cannot be explained by change in external temperature alone. An increase in counts for the same pixels in Fig. 3 would require a temperature change much greater than the change in external temperature would suggest from the data shown in Fig. 4. In addition, Fig. 4 highlights the possibility of using the pixels most responsive to temperature changes as dark current indicators. The pixel responses are predictable for temperature and exposure time, and the hottest pixels, pixels with the greatest dark current signal-to-noise ratio, can be used to predict and correct the dark count of other pixels on the imager.

\subsection{Canon PowerShot SD1000}

The SD1000, introduced 22 February 2007, has a 1/2.5-in. type CCD sensor (approximately $5.7 \times 4.3 \mathrm{~mm}$ ), with 7.1 million effective pixels in a grid of $3072 \times 2304$ pixels. To gauge the dark current response due to exposure time, we took ten images for each exposure time of 1, 5, 10, 20, 30, 40,50 , and $60 \mathrm{~s}$, with the 1 -s set taken first and increasing to $60 \mathrm{~s}$. The camera was set to an ISO setting of 400 and the external temperature within the enclosure was $24{ }^{\circ} \mathrm{C}$. The average was taken for each set of images after discard- 


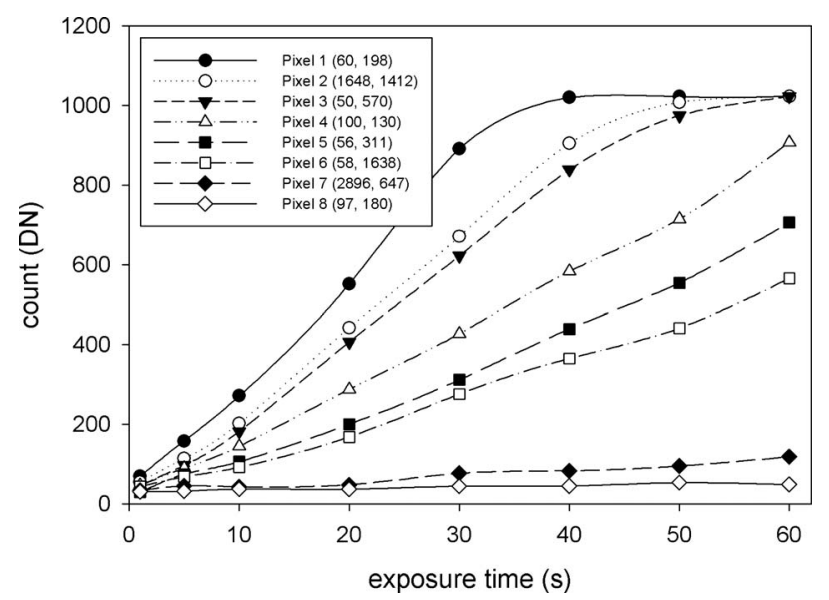

Fig. 5 Exposure time dependence of dark current for eight pixels taken at ISO $=400$ and $24^{\circ} \mathrm{C}$.

ing the maximum and minimum values for each pixel. Figure 5 shows the exposure time dependence for eight representative pixels on the imager. For each pixel, the pixel coordinates are given in parentheses. Pixels 1 through 6 are all hot pixels to varying degrees, with pixels 1 through 3 saturating within the taken regime and therefore not making ideal indicators for this range. Pixels 7 and 8 represent a more frequent pixel on the imager that has a smaller dark current. Characteristic of this type of common pixel is an increase in dark noise with increasing exposure time and a fairly linear response, except for when the pixel begins to approach the saturation level of 1023 counts.

Figure 6 shows the temperature dependence of dark current for the same eight pixels as displayed in Fig. 5. Again, each data point represents the average of ten frames, with the maximum and minimum values for each pixel removed. Dark current response versus temperature appears to be predictable until values near saturation for the pixels in the imager. Despite the claim by Canon that ISO sensitivity of the SD1000 goes up to 1600 , it was found that the dark current does not change past a setting of 800 and deviates from a linear dependence after a setting of 400. This ob-

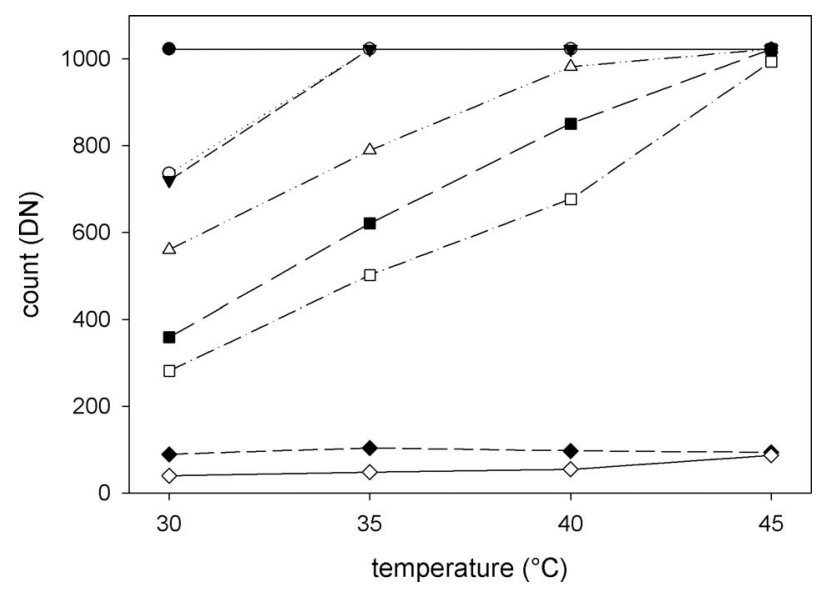

Fig. 6 Dependence of dark current for eight pixels on external temperature. Taken with ISO $=400$ and an exposure time of $50 \mathrm{~s}$. (Same pixels as shown in Fig. 5.)

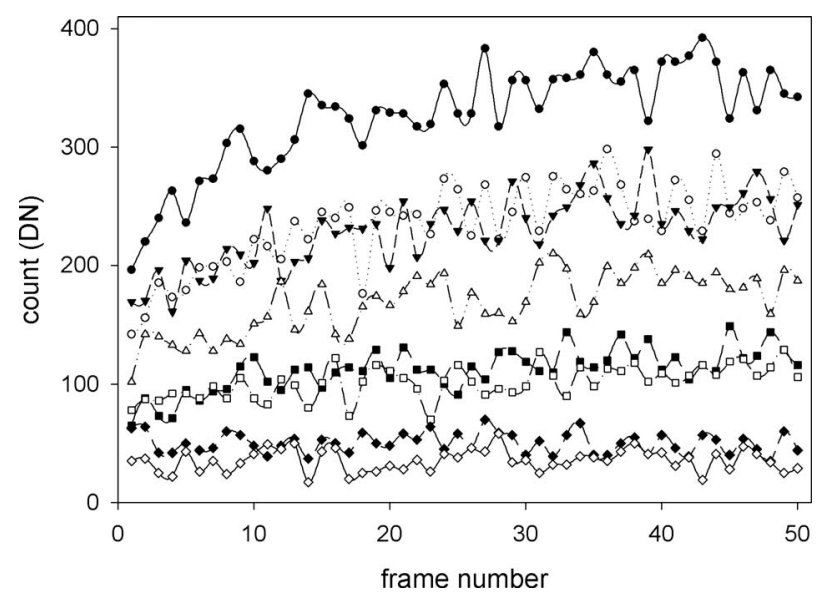

Fig. 7 Dark current dependence on frame numbers for eight pixels taken at ISO $=400,24^{\circ} \mathrm{C}$, and integration times of $15 \mathrm{~s}$, with a rest period of $60 \mathrm{~s}$ between frames. (Same pixels as shown in Fig. 5.)

served response may be due to additional processing done by the Canon software to obtain effective ISO sensitivities past 400 , therefore it is not seen in this data due to use of the CHDK software. Regardless, the dark current does show a linear and predictable response for most of the ISO range. Likewise, dark current response versus temperature appears to be predictable until values near saturation for the pixels in the imager, as shown for the eight pixels presented in Fig. 6.

To ascertain the effect of increasing frame number when multiple images were taken in succesion for compact cameras, a set of 50 frames were taken with the SD1000 at an ISO setting of 400, an integration time of $15 \mathrm{~s}$, and with a 60 -s rest between frames. The temperature within the enclosure increased from $23.8^{\circ} \mathrm{C}$ during the first frame to $24.3{ }^{\circ} \mathrm{C}$ during the last frame. Figure 7 shows the response of eight pixels on the imager with these settings. Even with a rest time four times greater than the integration time, the dark current increases significantly from frame 1 to frame 50. As an example, pixel 1 nearly doubled in count, increasing from 196 counts in the first frame to 342 counts in the last frame, a relative increase of $74 \%$. This increase likely cannot be explained by the external temperature change of $0.5^{\circ} \mathrm{C}$ alone.

\section{Method of Correction for Dark Current}

Our method of correction is dependent on finding the chip's hot pixels-pixels that have higher than average values of dark noise. These hot pixels are unique to every imager and arise due to impurities within the pixel. ${ }^{4}$ To find the hot pixels, a pixel is compared to its neighbors by using the following equation:

$\operatorname{hotpix}(x, y)=\sum_{i=-1}^{1} \sum_{j=-1}^{1} \operatorname{weight}(i, j) \cdot \operatorname{pixel}(x+i, y+j)$,

where $(x, y)$ are the coordinates of the pixel on the image, and $\operatorname{pixel}(x, y)$ is its dark count. The weight $(i, j)$ is chosen to be dependent on a neighbor's relative location to the pixel so that the sum of all weighting factors equals zero. Different weighting factors and numbers of neighbors can 


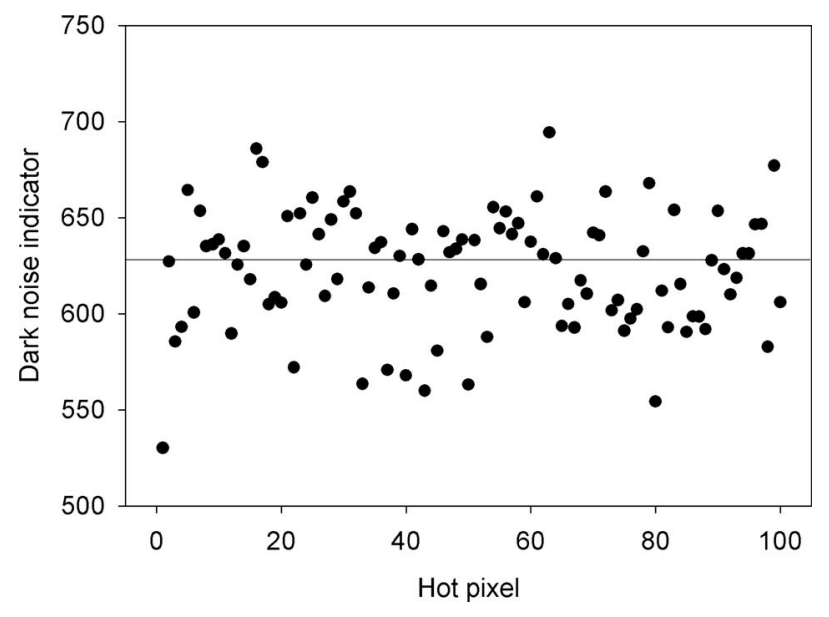

Fig. 8 Dark current indicators for a dark frame on the SD1000 taken at $30 \mathrm{~s}, \mathrm{ISO}=400$, and $24^{\circ} \mathrm{C}$.

be chosen. For the corrections done in this work, the center pixel was weighted with a value of 1 , the pixels on the corners were weighted with a value of -0.05 , and the neighbors directly adjacent were weighted with a value of -0.2 . If all neighboring pixels have comparatively similar values to the center pixel, the value for hotpix $(x, y)$ will be close to zero. However, if all neighboring pixels have comparatively smaller values, the value for hotpix $(x, y)$ will be large. Next, the values of hotpix $(x, y)$ are ranked and the $n$ hottest pixels are chosen. The average of hotpix $(x, y)$ for the $n$ hottest pixels is calculated and is used as a dark current indicator for the chip $D_{\text {ind }}$. $D_{\text {ind }}$ is dependent on the imager's temperature, the frame exposure time, and ISO setting as shown before; however, knowledge of the actual values for these parameters is not required and indeed not used to calculate $D_{\text {ind }}$. The values for hotpix $(x, y)$ are then fitted as a quadratic least squares function of the indicator, such that:

$\operatorname{hotpix}(x, y)=a \cdot D_{\text {ind }}^{2}+b \cdot D_{\text {ind }}+c$.

Pixels with poor fits are disregarded from the calculation. The values for the fitting parameters $a, b$, and $c$ can then be saved for the hottest pixels along with the coordinates of the hot pixels. The next step in the protocol is to determine the counts of all pixels with respect to $D_{\text {ind }}$. To accomplish this, the counts of each pixel for frames taken with different amounts of dark current are fitted with a quadratic least squares fit versus the dark current indicator. Since the fit is later used to calculate the dark count independently from the neighboring pixels, the actual count of the pixels (not the counts with respect to the neighboring pixels) is used to determine the fitting parameters. Therefore, three matrices are calculated and stored with unique $a, b$, and $c$ parameters for every pixel on the imager. These parameters can then be used to evaluate the relative amount of dark current in any image within the calibrated regime.

Figure 8 shows the dark noise indicator of a dark frame for 100 indicator pixels on the Canon PowerShot SD1000, as well as a line representing the median value of all the indicators. The number of indicator pixels chosen (100 in this instance) is a result of choosing the $n$ hottest pixels
(2500 in this case) and then filtering out pixels based on, among other things, quality of fit to the quadratic parameters. The dark noise indicated by all the indicator pixels is similar, but due to random noise there is a spread in the values for the indicator. Therefore, a median value is taken of all the indicators to determine a frame's $D_{\text {ind }}$ value. The median value can then be used in combination with the three parameter files to calculate the appropriate amount of dark current for every pixel on the imager. The advantage of using the median over the mean is not immediately apparent when calculating $D_{\text {ind }}$ values for a dark frame; however, when a light signal is also present on the imager, the difference between the mean and median values can be significant due to parts of the imager being exposed to relatively greater amounts of light, creating an artificially elevated $D_{\text {ind }}$ for any indicator pixels in those regions. Assuming an appropriately large enough number of chosen indicator pixels, the median value will be less affected by the light signal than the mean, and is therefore a better indicator of the dark noise.

It was shown that each imager had pixels that demonstrated predictable behavior on each imager to exposure time, gain, and temperature. These pixels could therefore be used to accurately predict the amount of dark current for all pixels on the imagers.

\section{Dark Current Correction}

We again present data for only one camera in each of the classes; however, similar results in correction were seen for the Nikon D70, Nikon D700, and the Canon EOS-20Da in the DSLR class, and for the other Canons (the A570 IS and the S2IS) in the compact consumer class.

\subsection{Canon EOS-20D}

To determine the success of correction by the built-in noise reduction mode on the Canon 20D, three dark frames were taken. The first frame was taken such that the noise reduction mode was turned on, and the second and third frame were taken with noise reduction turned to off. The third image was subtracted from the second to obtain a corrected frame. To test the relative success of correction, we calculated the standard deviation of the original frame and the corrected frames. In an ideal correction, subtraction of a second dark frame would yield a histogram with a narrow distribution around the value of zero, with only small deviations due to shot noise, the noise due to the statistical nature of the generation of dark current. A smaller standard deviation of the corrected dark frame would indicate a better correction than one with a larger standard deviation. In addition, a large standard deviation indicates a dark frame with large variations in the uniformity of dark noise. However, it is worth noting that using the standard deviation as the sole means of testing the quality of correction is not fully adequate, because it fails to take into account the effect that small numbers of outlier pixels with poor correction would have on the quality of the image. These pixels would have values greatly deviating from the dark noise in the original image, and therefore a correction would result in these pixels showing up as either darker than their surroundings due to an inaccurate overcorrection, or showing up as much brighter than the surroundings due to an inaccurate undercorrection. Ultimately these pixels do need to 


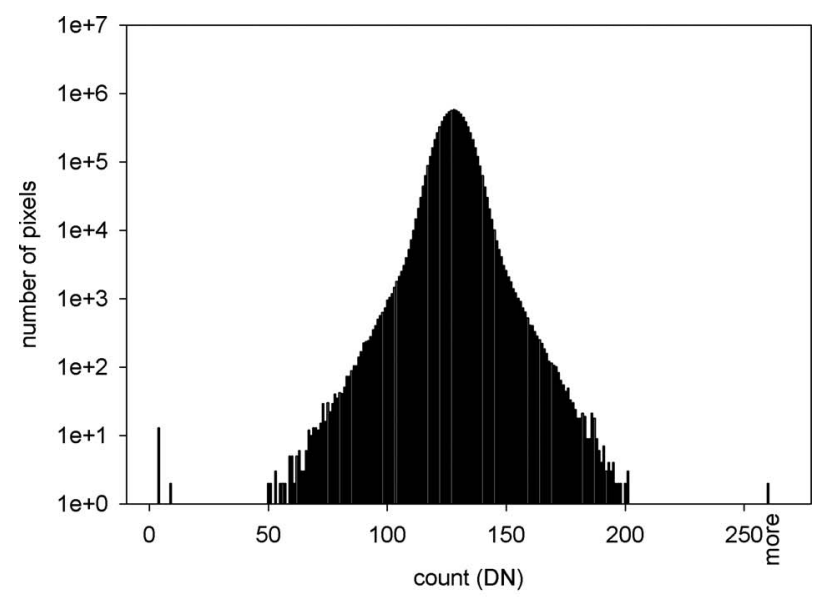

(a)

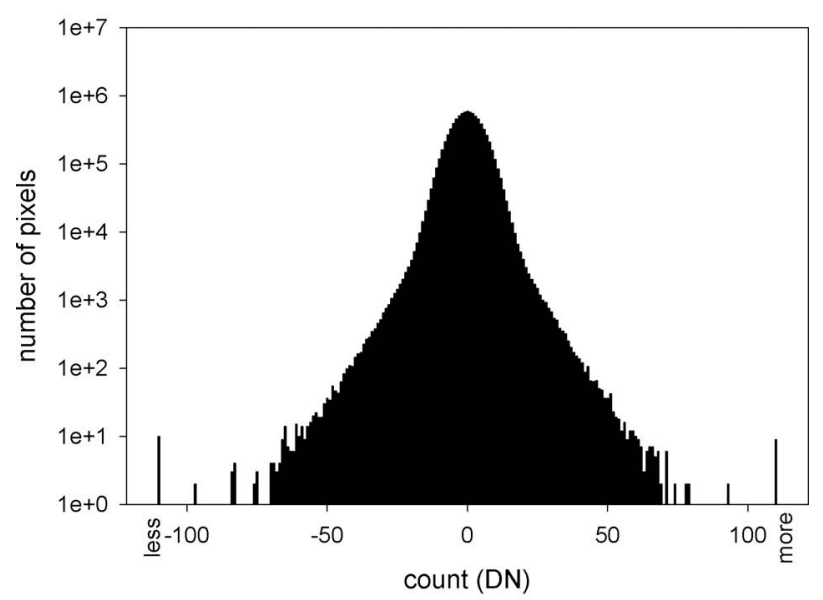

(b)

Fig. 9 Correction of a $30-\mathrm{s}, 800$ ISO setting, $25^{\circ} \mathrm{C}$ image. (a) Histogram of the frame taken with Canon 20D's noise reduction mode turned on. (b) Histogram of a corrected frame using another individual image for correction.

be properly corrected to obtain the most aesthetically pleasing image; however, the standard deviation provides an easily comparable value that can be used to weigh the success of correction. The histogram of the frame with the Canon 20D's noise reduction mode turned on, shown in Fig. 9(a), has a standard deviation of 6.07 counts. The histogram is centered on a positive value likely due to a shift in offset such that the would-be negative values lie above zero as an actual image cannot contain negative values. The histogram of the corrected frame using another dark frame for correction, shown in Fig. 9(b), has a standard deviation of 6.05 counts. The similar values of the standard deviations suggest that the Canon 20D does indeed take a second dark frame to subtract off the original image. Regardless, the Canon 20D's method of correction is shown to be no better than using an individual dark frame for subtraction as a method of correction.

To test the possibility of using the method of correction discussed at the beginning of this section on the Canon 20D, a series of 30 images, five each at exposure times of $5,10,15,20,25$, and $30 \mathrm{~s}$, were used to generate the coefficients of correction. This represents a total of $525 \mathrm{~s}$ of camera time. For comparison, we also used two other methods of correction, an averaged master frame and an individual dark frame. An averaged master frame was generated by taking the pixel by pixel average, with maximum and minimum values removed from the average, of nine 30 -s frames. These images were taken after the image to be corrected, and represent $270 \mathrm{~s}$ of camera time. Figure 10(a) shows the histogram of the uncorrected frame. Two peaks are apparent in the uncorrected frame, but are seen more distinctly in Fig. 10(c), the histogram of the calculated frame, and Fig. 10(e), the histogram of the master frame. The peaks can be seen more distinctly in the average and calculated frame due to longer observations and the corresponding reduction in random noise. These peaks are thought to be caused by specific types of impurities within the imager. ${ }^{8}$ The dark current in the uncorrected frame was removed by subtracting the frame generated by the three methods discussed. The original uncorrected image has a standard deviation of 15.36 counts. In Fig. 10(b), the histogram for the correction using another individual frame is shown. This histogram has a standard deviation of 12.44 counts. Figure 10(d) shows the histogram for the correction using the calculated frame that has a standard deviation of 9.31 counts. Figure 10(f) shows the corrected frame with a standard deviation of 9.28 counts. Thus the correction using the calculated frame has a $39 \%$ decrease in standard deviation compared to the original frame, shows a similar improvement as an averaged frame, and improves on the correction using an individual frame.

Once the coefficients are calculated for an imager, dark frames can be quickly calculated for any exposure time within the calibrated region. A $15-\mathrm{s}$ frame taken with an ISO setting of 1600 and at a temperature of $23{ }^{\circ} \mathrm{C}$ was corrected using the method of calculation. The same coefficients that were calculated for the 30-s image were used to calculate this correction frame. The histogram for the original uncorrected image has a standard deviation of 11.59 counts. The correction using an individual frame has a standard deviation of 9.56 counts, whereas correction using the calculated frame creates a histogram with a standard deviation of 7.05 counts. The calculated frame decreases the standard deviation of the original image by $39 \%$ and again improves on the correction using a single image. Similar corrections were done using calculated frames for other exposure times within the calibrated region, and improvements were seen over the entire region compared to the original frame and single frame correction.

It remains to be shown whether a series of dark frames taken at different temperatures can accurately predict and correct for dark current in an image taken at a given temperature and exposure time within the calibrated range. Images were taken at external temperatures of 24, 30, 35, 40, and $45^{\circ} \mathrm{C}$. Five frames at exposure times of $5,10,15,20$, 25 , and $30 \mathrm{~s}$ at each temperature were used to generate the coefficients of correction. This represents a total of 150 images and $2625 \mathrm{~s}$ of camera time. A calculated frame was generated for a dark frame taken for $20 \mathrm{~s}$ at $30{ }^{\circ} \mathrm{C}$ with an ISO setting of 800 . The original uncorrected dark frame has a histogram with a standard deviation of 10.09 counts. For comparison, an individual frame, taken under the same conditions as the original image, was used for correction, and the resulting corrected frame's histogram is shown in Fig. 


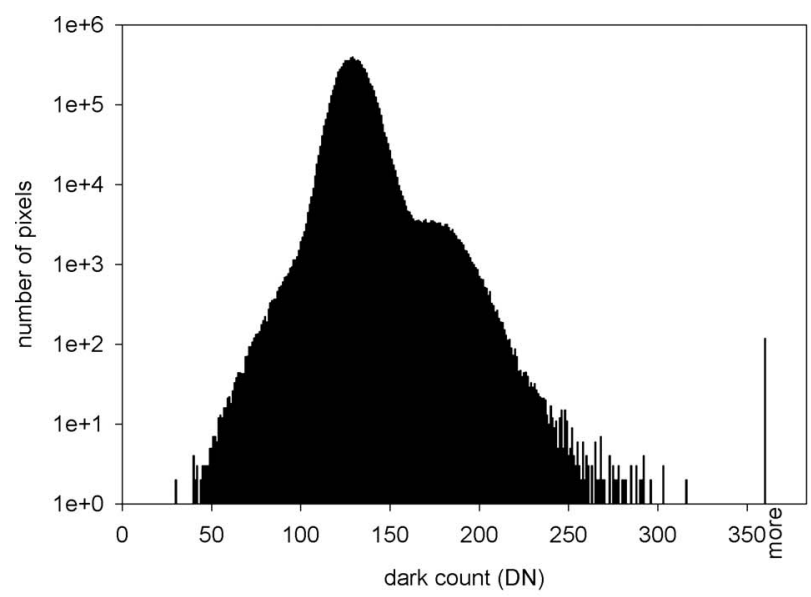

(a)

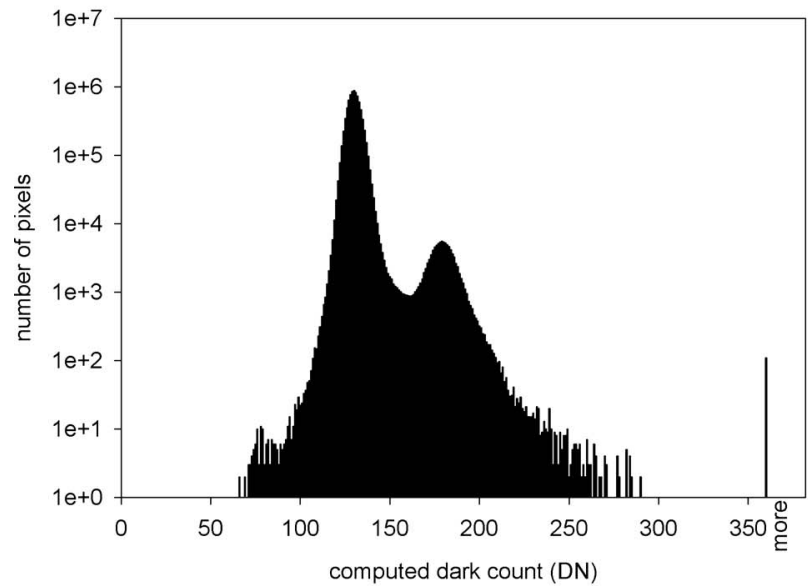

(c)

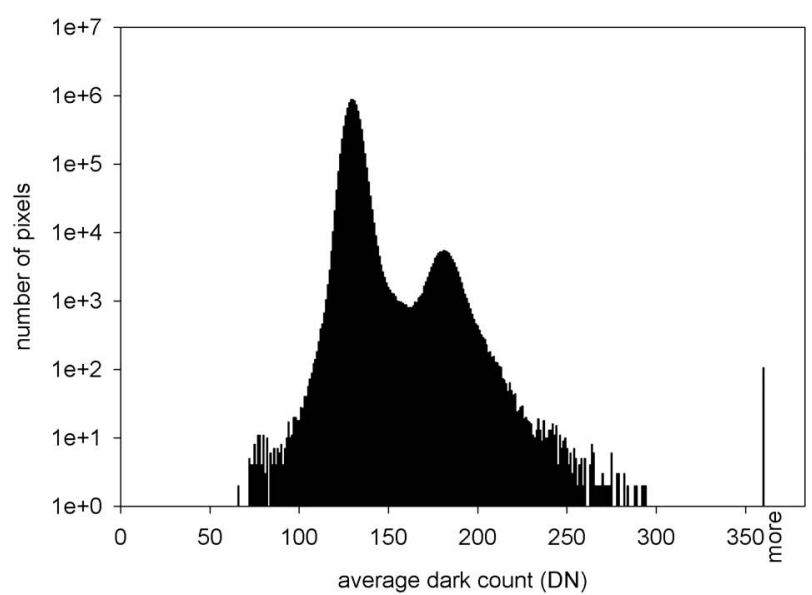

(e)

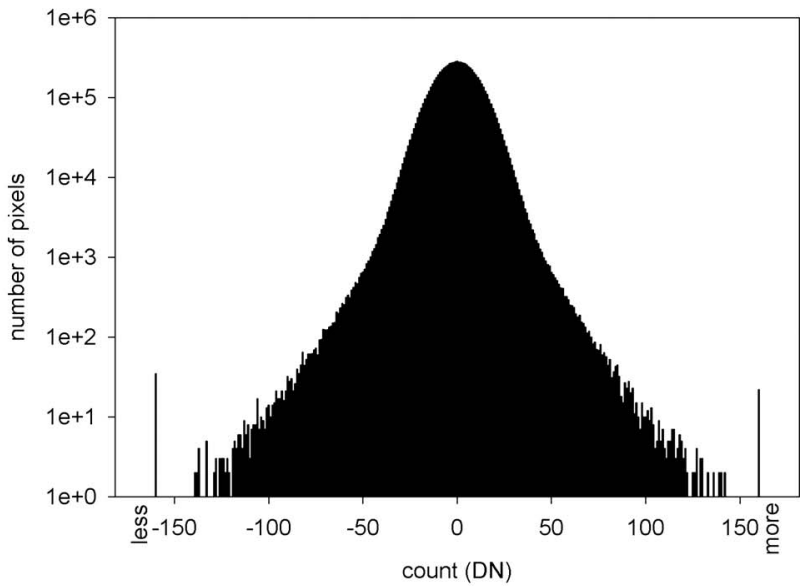

(b)

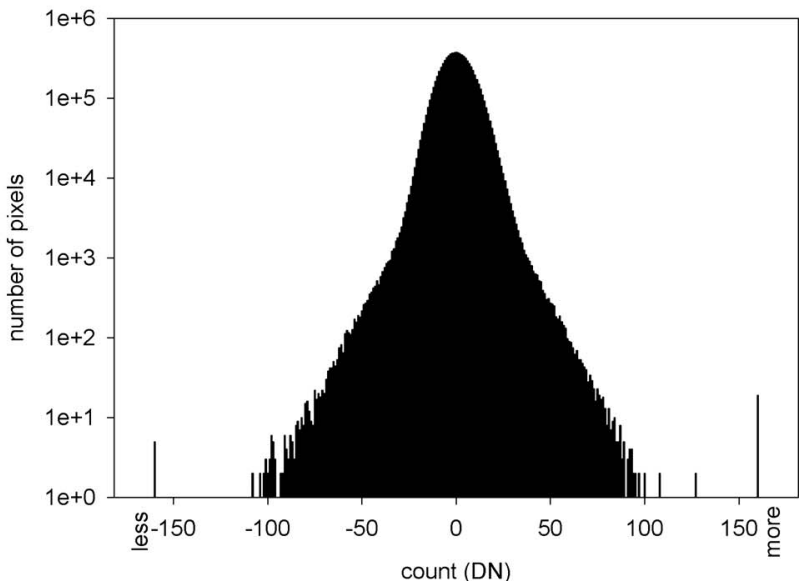

(d)

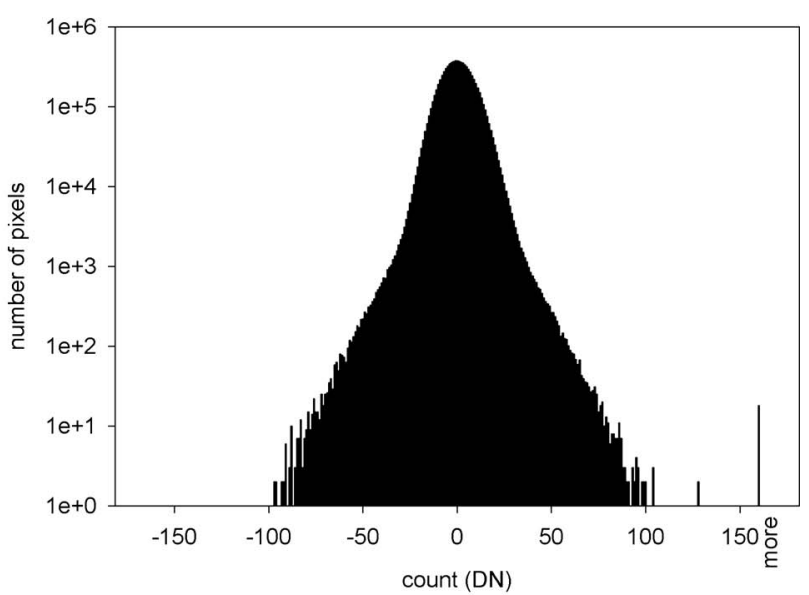

(f)

Fig. 10 Correction of a $30-\mathrm{s}, \mathrm{ISO}=1600,23^{\circ} \mathrm{C}$ image. (a) Histogram of the uncorrected frame. (b) Histogram of the corrected frame using an individual frame for correction. (c) Histogram of the constructed frame. (d) Histogram of the corrected frame using the constructed frame for correction. (e) Histogram of the master frame. (f) Histogram of the corrected frame using the averaged frame for correction.

11(a). This resulting histogram has a standard deviation of 7.09 counts. Figure 11(b) shows the corrected frame's histogram using the calculated frame for correction. This histogram has a standard deviation of 5.14 counts, an im- provement over using an individual frame, and is $49 \%$ smaller than the standard deviation of the original frame.

The generation of averaged master frames allows for more accurate correction than a correction involving an in- 


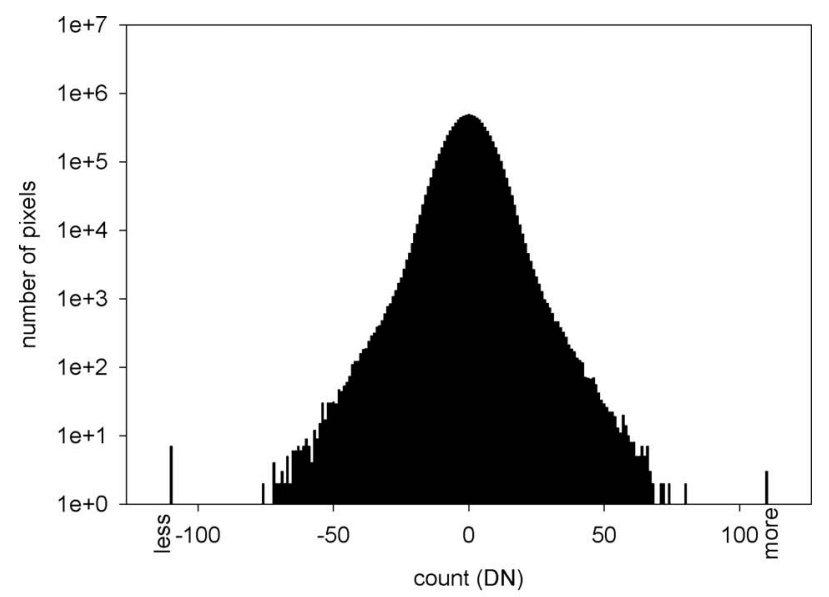

(a)

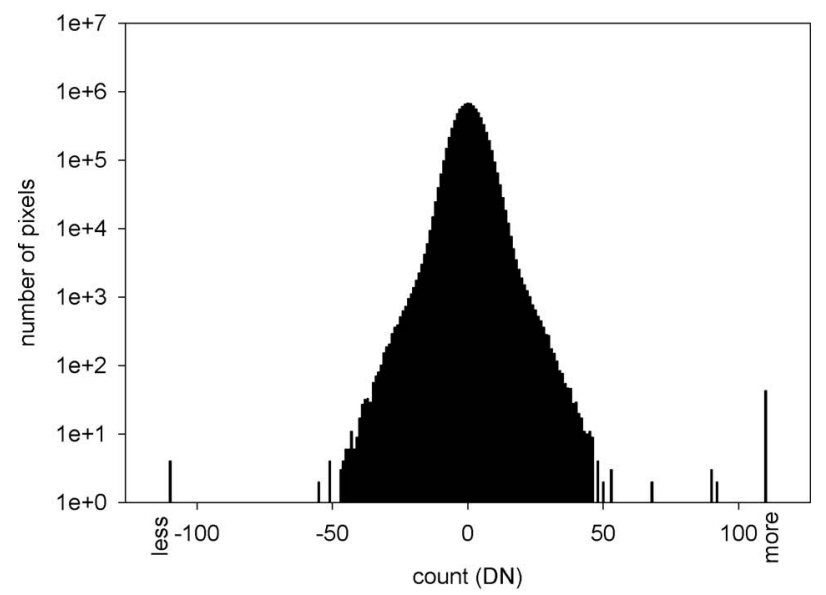

(b)

Fig. 11 Correction of a $20-\mathrm{s}$, ISO $=800,30^{\circ} \mathrm{C}$ image. (a) Histogram of the corrected frame using another individual frame. (b) Histogram of the corrected frame using the computed frame.

dividual frame, as demonstrated before. However, the master frame requires significantly more camera time than an individual frame, and is only accurate if the images composing the master frame are taken under the identical conditions as the image to be corrected. Figure 12(a) shows a histogram for the correction of the one hundredth image taken in the series of images used to generate Fig. 3, using an average master frame generated from the first ten images in the series, with the highest and lowest value for each pixel removed from the average. As noted previously, the external temperature within the enclosure increased from 24.0 to $25.2{ }^{\circ} \mathrm{C}$ during this run; however, the temperature of the sensor likely increased by significantly more. The increase in temperature of the sensor from the first ten frames to the one hundredth results in the bulge seen on the positive side of the histogram's peak. Due to many pixels having more dark current in the one hundredth frame than the first ten, a subtraction of the master frame results in these pixels having a positive value after the correction. Improvement in the standard deviation of the histogram is still seen; before correction, the histogram of the image has a standard deviation of 12.91 counts, and after correction the histogram has a standard deviation of 8.08 counts. Fig-

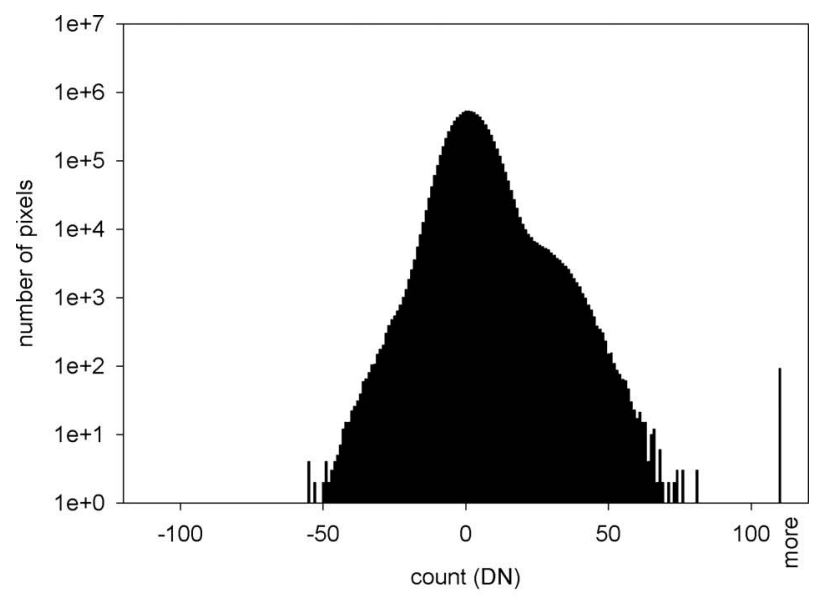

(a)

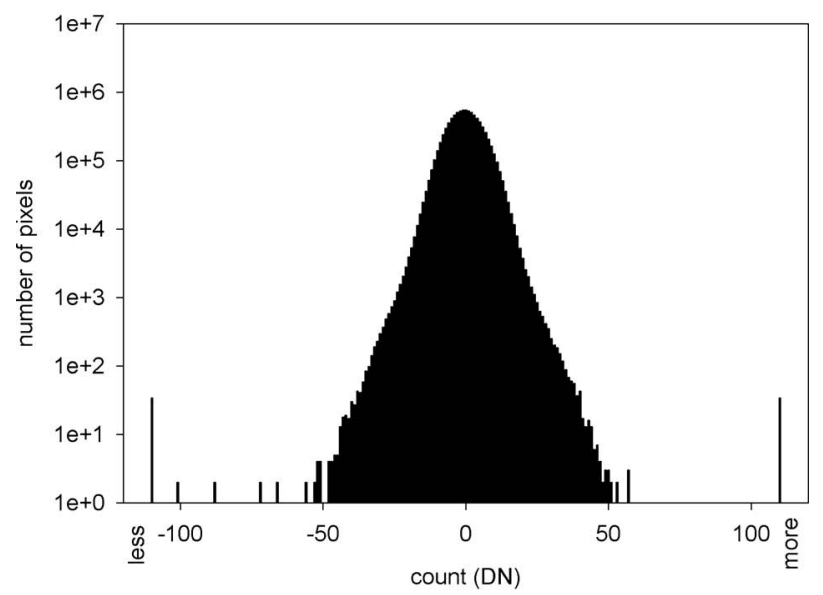

(b)

Fig. 12 Correction of a $30-\mathrm{s}$, ISO $=800,25^{\circ} \mathrm{C}$ image. (a) Histogram of the corrected frame using an averaged master frame taken at an external temperature of $24^{\circ} \mathrm{C}$. (b) Histogram of the corrected frame using the computed frame.

ure 12(b) shows a histogram of a corrected frame using a calculated frame from the coefficients for the same fitting as the previous correction. Using this method, the corrected frame has a standard deviation of 6.55 counts, a decrease of $49 \%$ compared to the original frame, and an improvement over the master frame correction. Small temperature fluctuations of $1{ }^{\circ} \mathrm{C}$ would not be uncommon over a session of image taking and, as shown in Sec. 2, the internal temperature of the imager itself likely changes by more for a long run of images. Therefore, a master frame generated from dark frames taken before or after many other frames will not provide accurate correction. The computed frames show better results, since the dark count is calculated based on the actual chip temperature.

\subsection{Canon PowerShot SD1000}

To test the possibility of dark noise correction in the SD1000 using hot pixels as indicators of dark current in an image, we took three dark frames each at $24{ }^{\circ} \mathrm{C}$, with an ISO setting of 400 , at $1,5,10,20,30,40,50$, and 60 -s exposure times, representing a total of $648 \mathrm{~s}$ of camera time. These frames were used to generate the coefficients of 


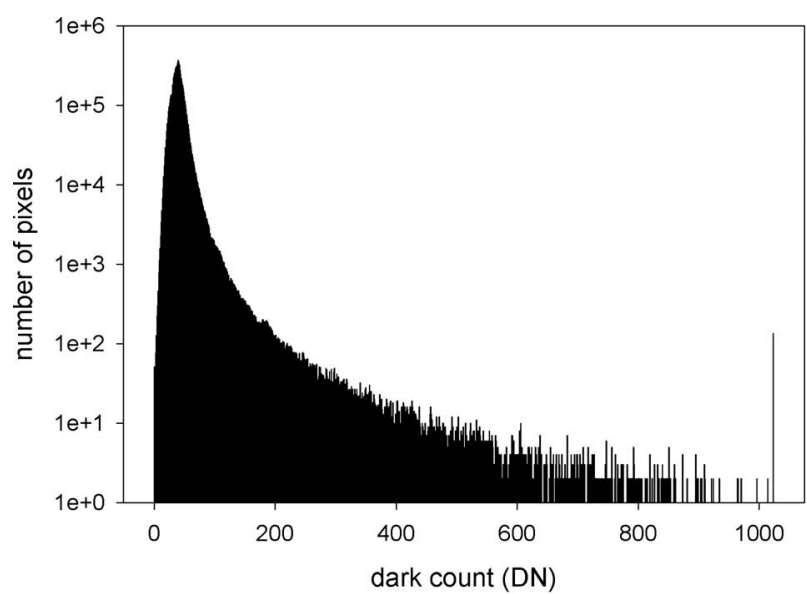

(a)

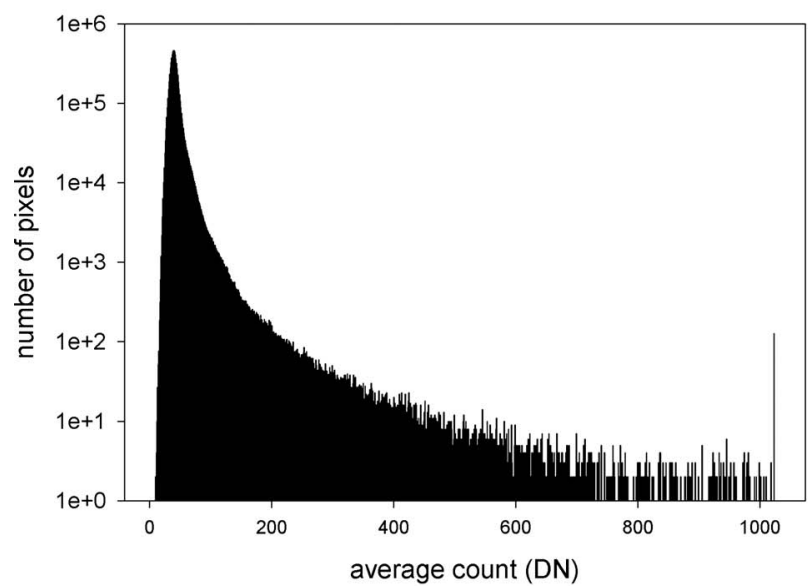

(c)

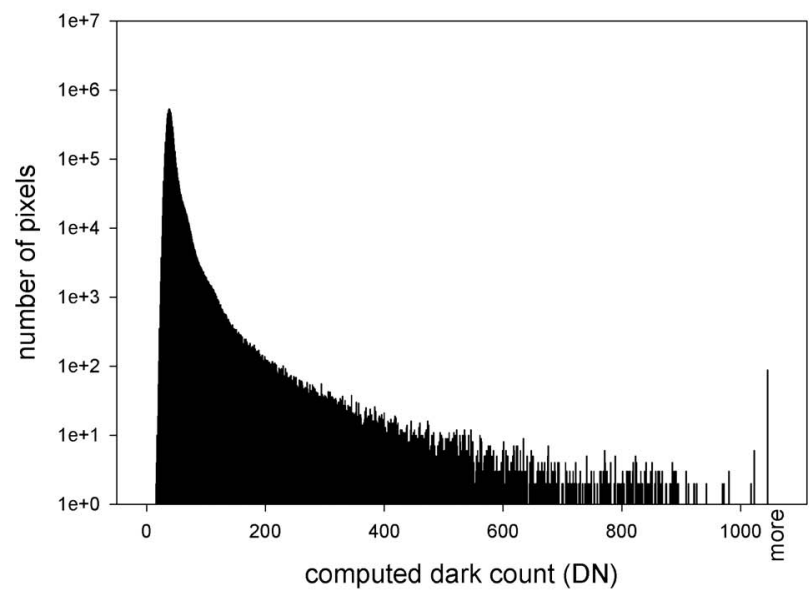

(e)

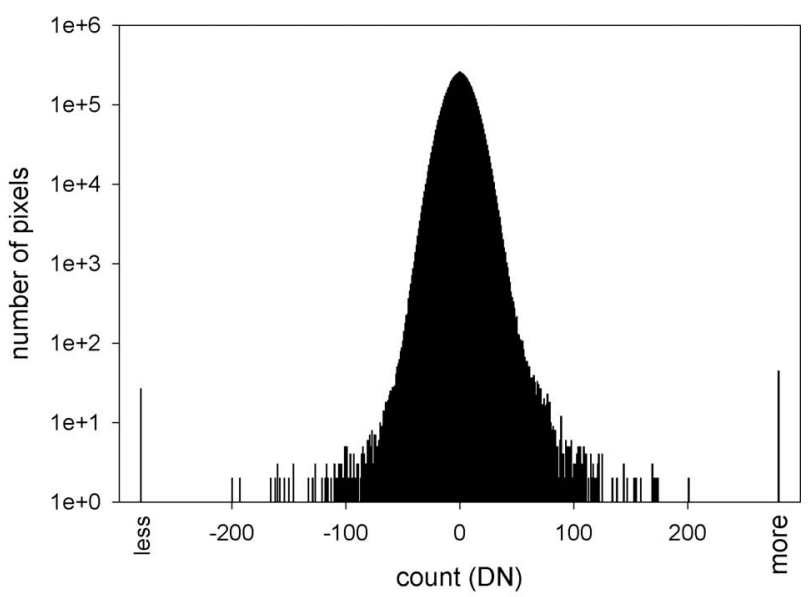

(b)

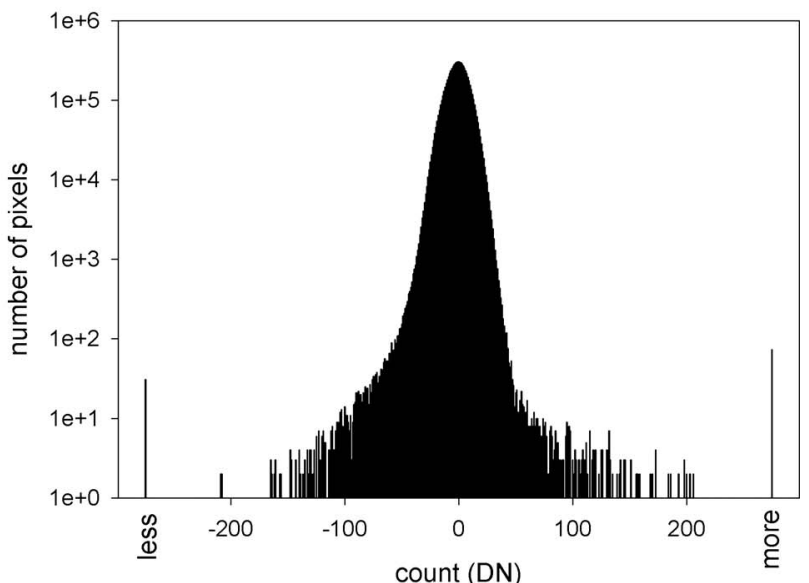

(d)

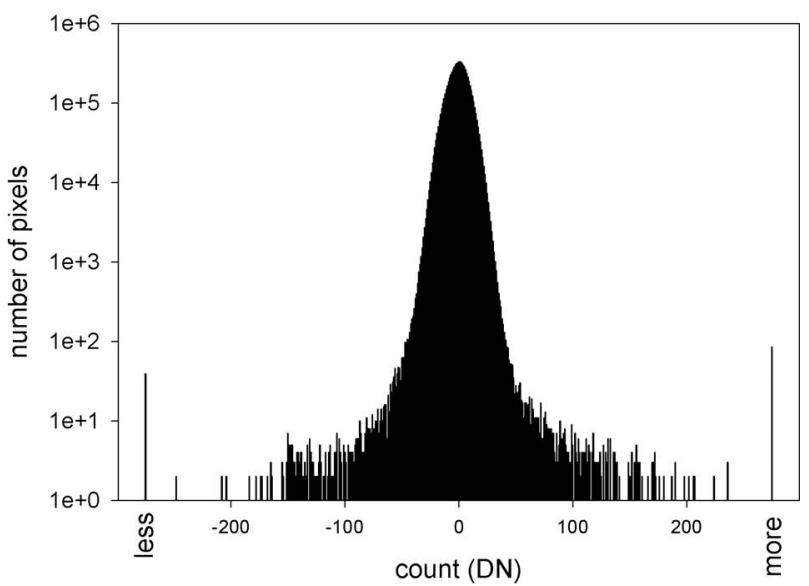

(f)

Fig. 13 Correction of a $30-\mathrm{s}$, ISO $=400,24^{\circ} \mathrm{C}$ image. (a) Histogram of the uncorrected frame. (b) Histogram of the corrected frame using an individual frame for correction. (c) Histogram of the master frame. (d) Histogram of the corrected frame using the master frame for correction. (e) Histogram of the computed frame. (f) Histogram of the corrected frame using the computed frame for correction.

correction. A computed dark frame was generated for a dark frame taken with an exposure time of $30 \mathrm{~s}$ taken at $24^{\circ} \mathrm{C}$, and with an ISO setting of 400 . Correction was done by subtracting the original image from the computed image. For comparison, we also used for subtraction both a single dark frame and an averaged master frame using four images. The averaged master frame was generated by taking a pixel by pixel average of the four frames, with the maximum and minimum values removed from the average. Shown in Fig. 13 are the histograms for the original image 


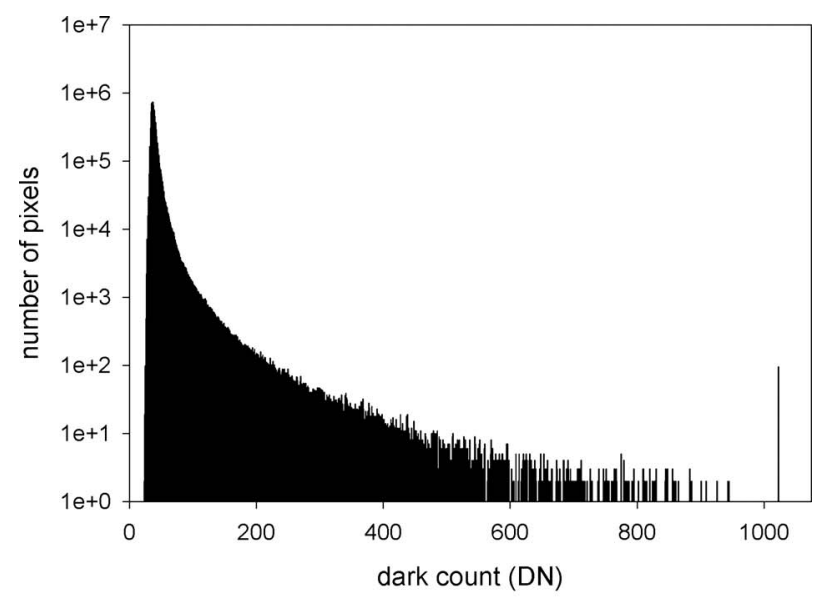

(a)

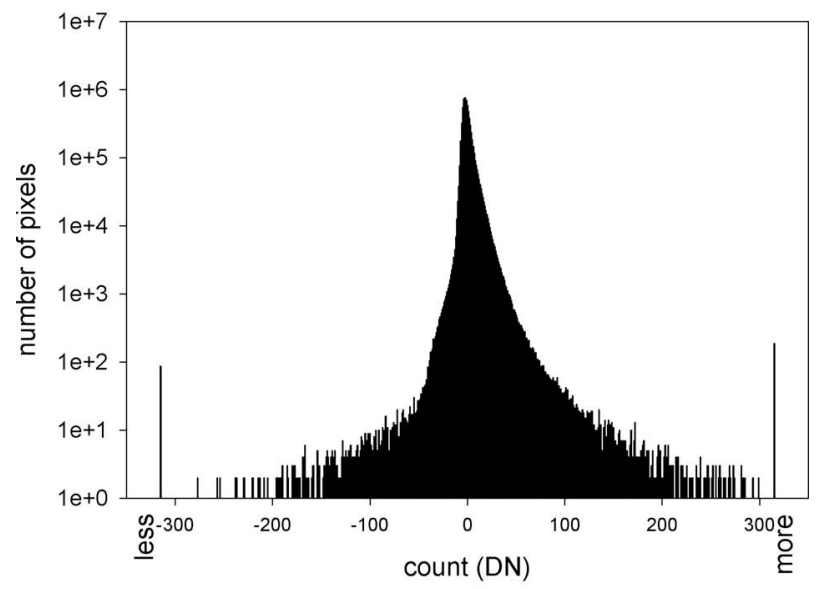

(b)

Fig. 14 Correction of a $64-\mathrm{s}$, ISO $=100,36^{\circ} \mathrm{C}$ image. (a) Histogram of the uncorrected frame. (b) Histogram of the corrected frame using a computed frame for correction.

[Fig. 13(a)], the corrected frame after subtraction of a single dark frame [Fig. 13(b)], the averaged master frame [Fig. 13(c)], the corrected frame after subtraction of the averaged master frame [Fig. 13(d)], the computed dark frame [Fig. 13(e)], and the corrected frame after subtraction of the computed frame [Fig. 13(f)].

The histogram of the uncorrected frame shows a single sharp peak with low dark current values and a steady decrease in frequency of pixels at higher dark current values. This trend continues until saturation with 135 pixels reading saturation. This histogram has a standard deviation of 18.6 counts. Standard deviations of the corrected frames are 12.0, 10.1, and 9.5 counts for corrections using the single frame, the averaged master frame, and the computed frame, respectively. Thus the correction using the computed frame has a $49 \%$ decrease in the standard deviation compared to the original, and shows an improvement over the use of both the single frame or the averaged master frame corrections.

An advantage of using the computational method of correction is that it does not require dark frames to be taken at the same time as the original image with the same exact settings. To show that the computational method can work on dark frames not taken with any of the same settings as the dark frames used to generate the coefficients of correction, a computed frame was generated for a dark frame taken at $64 \mathrm{~s}$ with an ISO setting of 100 and at an external temperature of $36{ }^{\circ} \mathrm{C}$. The histogram for the original frame has a standard deviation of 16.8 counts and is displayed in Fig. 14(a). The histogram for the corrected frame after subtraction of the computed frame is shown in Fig. 14(b). This histogram has a standard deviation of 7.8 counts, a decrease of $54 \%$ from the original.

\section{Summary}

Dark current within consumer cameras has been shown to vary with exposure time, temperature, and ISO setting. In addition, operation of the camera itself has been shown to alter the amount of dark current in subsequent frames, likely due to an increase in temperature of the imager itself from heat generated by internal camera circuitry. Correction for dark current has been shown to be successful despite this added complexity. Correction using single dark frames, averaged master frames, and computed dark frames using hot pixels as dark current indicators show improvement in the levels of noise compared to the original frames. In particular, the method of using past dark current behavior and hot pixels on the imager as indicators to compute dark frames has been shown to be comparable, and at times better, than the use of an averaged master frame, as well as being consistently more accurate than the use of a single dark frame, the apparent method of choice designed to be used by most consumer cameras. For the seven cameras we analyzed, corrections using computed frames show a 40 to $72 \%$ decrease in the amount of noise compared to the originals. Computational dark frame correction also has the benefit of being possible at any time after an image is taken, and does not require dark frames taken at the identical temperature, ISO setting, and exposure time as the original.

\section{References}

1. R. Widenhorn, A. Rest, M. M. Blouke, R. L. Berry, and E. Bodegom, "Computation of dark frames in digital imagers," Proc. SPIE $\mathbf{6 5 0 1}$ 650103 (2007)

2. W. C. Porter, B. Kopp, J. C. Dunlap, R. Widenhorn, and E. Bodegom, "Dark current measurements in a CMOS imager," Proc. SPIE 6816, 68160C (2008)

3. S. M. Sze, Physics of Semiconductor Devices, John Wiley and Sons, New York (1967).

4. R. D. McGrath, J. Doty, G. Lupino, G. Ricker, and J. Vallerga, "Counting of deep-level traps using a charge-coupled device," IEEE Trans. Electron Devices 34, 2555 (1987).

5. W. C. McColgin, J. P. Lavine, J. Kyan, D. N. Nichols, and C. V. Stancampiano, "Dark current quantization in CCD image sensors," Intl. Electron. Device Meet. 113, 13-16 (1992).

6. CHDK Wiki members, "CHDK Wiki," see http://chdk.wikia.com/ wiki/CHDK.

7. C. Buil, "Comparative test Canon 10D/ Nikon D70 in the field of deep-sky astronomy," see http://astrosurf.com/buil/d70v10d/ eval.htm.

8. W. C. McColgin, J. P. Lavine, and C. V. Stancampiano, "Probing metal defects in CCD image sensors," Mater. Res. Soc. Symp. Proc. 378, 713 (1995). 


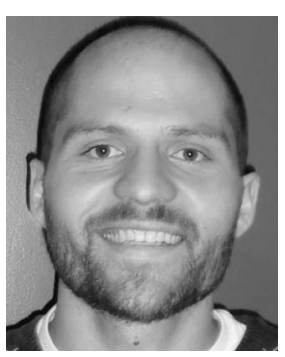

Justin C. Dunlap received his BA degree from the University of California, Berkeley, in physics and astrophysics in 2002 . He received his MS degree from Portland State University in physics in 2009. He is currently a graduate student at Portland State University continuing to research dark current and digital imagers.

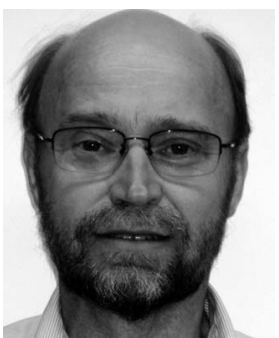

Erik Bodegom completed his engineering degree at Technical University Delft (Netherlands) and his $\mathrm{PhD}$ (1982) at Catholic University of America in Washington, D.C. Since 1985 he has been with Portland State University, where he is a professor and Chair of the Department of Physics. His pedagogical activities have included the writing of a set of astronomical exercises, the development of a resistance probe for use in schools, and the development of software to remotely control observatories. He has served as the president of the Oregon Academy of Science. His research activities have ranged from low temperature physics to statistical mechanics and solid-state physics. Over the last decade, his work has focused on the use and understanding of digital imagers.

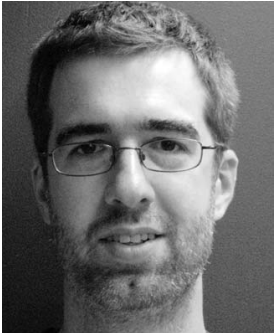

Ralf Widenhorn received his Vordiplom in physics from University of Konstanz in 1997, his MS in physics in 2000, and his Doctorate in environmental sciences and resources/physics in 2005, both from Portland State University. He has published numerous papers on digital imagers and is currently a researcher and professor at Portland State University. Some of his work is available in a book entitled Chargecoupled Devices-Performance and Dark Noise Characteristics from VDM Verlag. 